\title{
Video-assisted thoracoscopic lobectomy with an anomalous pulmonary vein
}

Ryota Sumitomo, MD, Takamasa Fukui, MD, Yosuke Otake, MD, PhD, and Cheng-long Huang, MD, PhD, Osaka, Japan

From the Department of Thoracic Surgery, Tazuke Kofukai Medical Research Institute, Kitano Hospital, Osaka, Japan.

Disclosures: Authors have nothing to disclose with regard to commercial support.

Received for publication April 12, 2016; revisions received May 10, 2016; accepted for publication July 24, 2016; available ahead of print Sept 17, 2016

Address for reprints: Ryota Sumitomo, MD, Department of Thoracic Surgery, Tazuke Kofukai Medical Research Institute, Kitano Hospital, 2-4-20 Ohgimachi, Kita-ku, Osaka 530-8480, Japan (E-mail: rt-sumitomo@ kitano-hp.or.jp).

J Thorac Cardiovasc Surg 2016;152:1398-9

$0022-5223 / \$ 36.00$

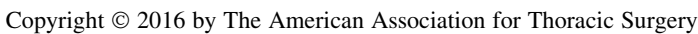

http://dx.doi.org/10.1016/j.jtcvs.2016.07.075

\begin{tabular}{|c|c|}
\hline $\begin{array}{l}\text { FCS } \\
\text { VIDEO } \\
\text { CLIIP }\end{array}$ & Video clip is available online. \\
\hline
\end{tabular}

Anomalous vessels are sometimes found during surgery. Subotich and colleagues ${ }^{1}$ reported that $16.4 \%$ of patients had pulmonary vessel variations found during lung resection. Transposition of the right pulmonary artery (PA) and the V1 through V3 segments of the pulmonary vein, however, is extremely rare.

\section{CLINICAL SUMMARY}

A 54-year-old man with suspected lung cancer was referred to our hospital. Computed tomography showed a $15-\mathrm{mm}$ diameter tumor in the right upper lobe that was strongly suspected to be malignant. No lymph node metastases or distant metastases were detected on positron emission tomography or magnetic resonance imaging of the brain. A transbronchial lung biopsy was not performed because of the peripheral location of the tumor.

During the preoperative evaluation, we reconstructed 3-dimensional (3D) models of the pulmonary vessels and bronchial trees from computed tomographic images with the Fujifilm Synapse Vincent system (Fujifilm Corporation, Tokyo, Japan). The 3D models revealed anomalous V1 through V3 segments that passed between the PA and bronchus (Figure 1). They also showed an ectopic B2 and tracheal bronchus.

Video-assisted thoracoscopic surgery (VATS) was performed. Wedge resection for the upper-lobe tumor was performed first, and it was diagnosed as lung cancer by intraoperative evaluation of a frozen section. Then, upper lobectomy with VATS was performed. The anterior trunk of the PA was first cut by an endostapler. After a minor fissure was divided, the anomalous V1 through V3

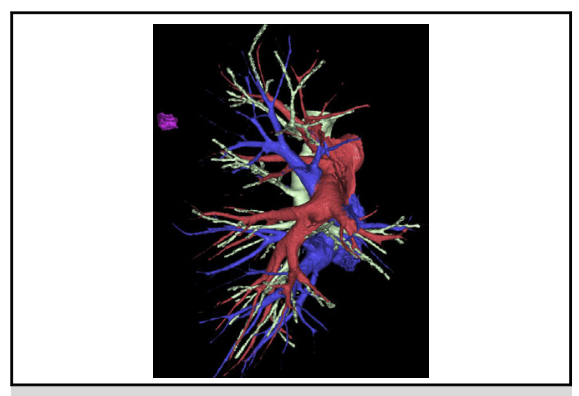

Preoperative 3D CT showed an anomalous V1-V3 segment passing between the PA and bronchus.

\section{Central Message}

Three-dimensional imaging enabled lobectomy despite an anomalous vein of the right upper lobe passing between the artery and bronchus.

See Editorial Commentary page 1400.

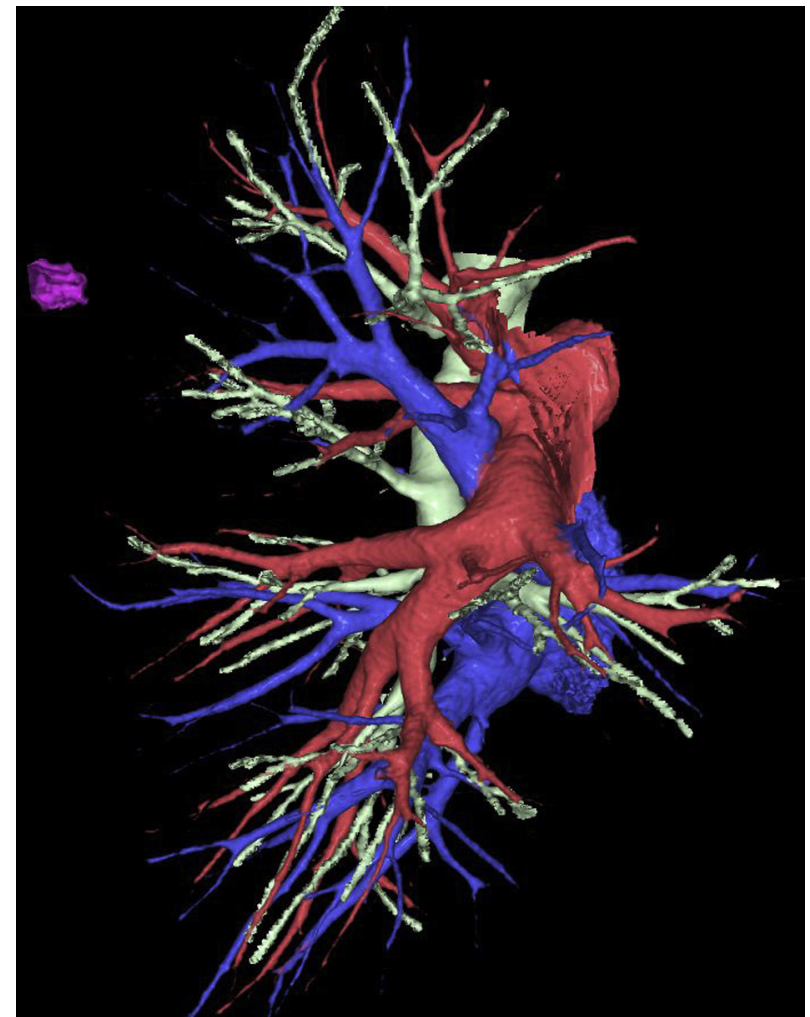

FIGURE 1. A preoperative 3-dimensional image shows anomalous V1 through V3 segments of the right pulmonary vein passing between the pulmonary artery and bronchus. 


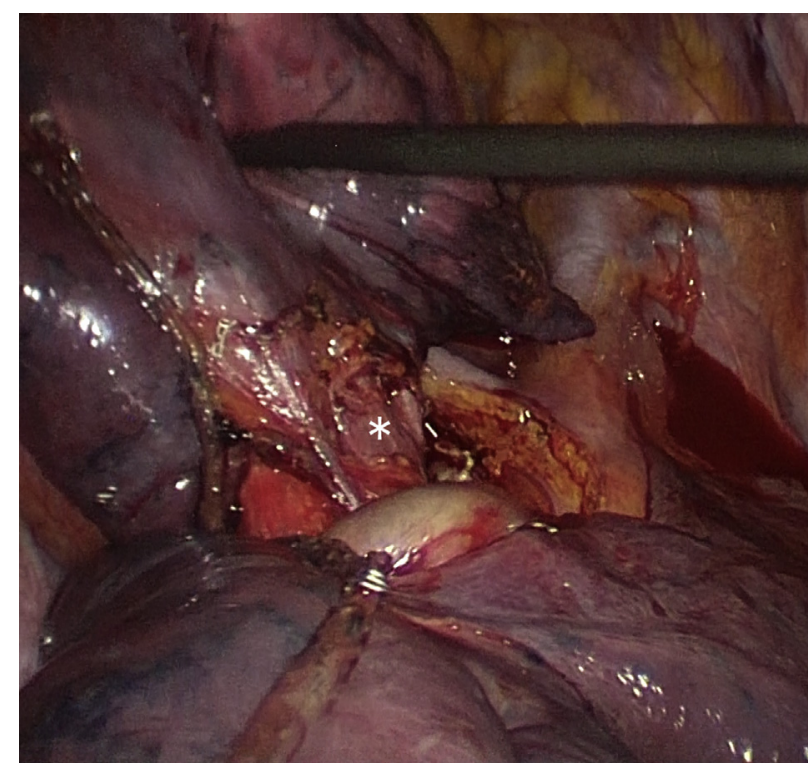

FIGURE 2. Intraoperative findings. The asterisk shows the V1 through V3 segments of the right pulmonary vein passing between the pulmonary artery and bronchus.

segments were found, but they were unable to be dissected at that time. An A2 segment, which branched through the superior and posterior side of the anomalous V1 through V3 segment, was therefore carefully dissected. Subsequently, the posterior part of a major fissure, between $\mathrm{S} 2$ and S6, was divided. At that point, the anomalous V1 through V3 segments were easily dissected and cut by an endostapler (Figure 2 and Video 1). The A2 segment was then cut by an endostapler. Finally, the ectopic B2 and tracheal bronchus were cut with endostaplers, and the right upper lobectomy was completed. Mediastinal and hilar lymph node dissections were also performed.

The tumor was diagnosed as adenocarcinoma, with a pathologic stage of T2a N0 M0 because of its pleural invasion. The chest tube was removed on the first postoperative day, and the patient's postoperative course was stable.

\section{DISCUSSION}

The V1 through V3 segments of the right pulmonary vein are usually located at the front of the main PA. In this case, the anomalous V1 through V3 segment was not found until the anterior trunk of the PA, and the major and minor fissures were divided. It would have been difficult to identify the superior pulmonary vein during surgery had this anomaly not been detected preoperatively. The 3D imaging showed an anomalous V1 through V3 segment passing between the PA and bronchus, which was the transposition of the right PA and the V1 through V3 segments. Without this preoperative information, the lobectomy would have been difficult to perform safely, and critical injury of the right main PA might have occurred while trying to locate the V1 through V3 segments at the front of the hilum.

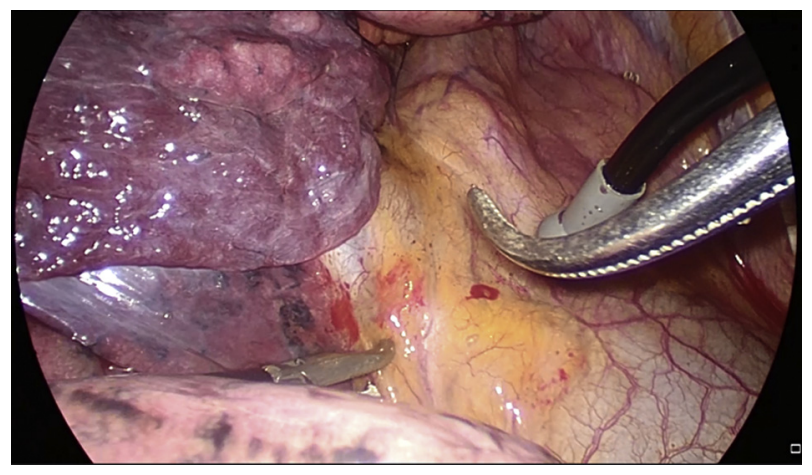

VIDEO 1. The anterior trunk of the pulmonary artery was first cut by an endostapler. After a minor fissure was divided, the anomalous V1 through V3 segments were found, but they were unable to be dissected at that time. An A2 segment was carefully dissected, which branched through the superior and posterior side of the anomalous V1 through V3 segments. Subsequently, the posterior part of a major fissure, between the S2 and S6 segments, was divided. At that point, the anomalous V1 through V3 segments were easily dissected and cut by an endostapler. The A2 segment was then cut by an endostapler. Video available at: http://www. jtcvsonline.org/article/S0022-5223(16)31044-3/addons.

The Synapse Vincent software is more useful and less invasive than conventional pulmonary angiography. ${ }^{2} \mathrm{~A}$ surgeon without expert knowledge of synthetic imaging can reconstruct 3D images of each patient quickly and easily. The 3D imaging allows the user to rotate the objects freely and change the images' dimensions. Surgeons thus can obtain more information about the positional relationship of the pulmonary vasculature and the tracheobronchial tree, which is impossible with conventional angiography.

VATS has been accepted as a common operation for lung cancer. ${ }^{3}$ It is less invasive and is useful for visualizing structural details. Unlike with thoracotomy, however, it is impossible to see the whole lung or to touch the vessels and tumor during VATS. This disadvantage could cause a misunderstanding of vessel abnormalities during surgery, which could in turn result in vessel injury. The use of 3D imaging thus can enable VATS lobectomy to be performed safely because it shows the structure of the pulmonary vessels and bronchi preoperatively.

The 3D imaging software was very useful in guiding the operation in this patient. It remains to be seen how often the imaging described here might affect the strategy and outcome of resection for future patients.

\section{References}

1. Subotich D, Mandarich D, Milisavljevich M, Filipovich B, Nikolich V. Variations of pulmonary vessels: some practical implications for lung resections. Clin Anat. 2009;22:698-705.

2. Ikeda N, Yoshimura A, Hagiwara M, Akata S, Saji H. Three dimensiona computed tomography lung modeling is useful in simulation and navigation of lung cancer surgery. Ann Thorac Cardiovasc Surg. 2013;19:1-5.

3. Yan TD, Cao C, D’Amico TA, Demmy TL, He J, Hansen H, et al. Video-assisted thoracoscopic surgery lobectomy at 20 years: a consensus statement. Eur J Cardiothorac Surg. 2014;45:633-9. 